\title{
Utility of three-dimensional printing in preoperative planning for children with anomalous pulmonary venous connection: a single center experience
}

\author{
Jiajun Xu ${ }^{1,2}$, Yangfan Tian ${ }^{1}$, Jun Yin ${ }^{2}$, Jinhua Wang ${ }^{1}$, Weize Xu ${ }^{1}$, Zhuo Shi ${ }^{1}$, Jianzhong Fu ${ }^{2}$, Qiang Shu ${ }^{1}$ \\ ${ }^{1}$ Department of Heart Center, The Children's Hospital, School of Medicine, Zhejiang University, Hangzhou 310058, China; ${ }^{2}$ Key Laboratory of 3D \\ Printing Process and Equipment of Zhejiang Province, School of Mechanical Engineering, Zhejiang University, Hangzhou 310058, China
}

Correspondence to: Qiang Shu, PhD. Department of Heart Center, The Children's Hospital, School of Medicine, Zhejiang University, Hangzhou 310058, China. Email: shuqiang@zju.edu.cn.

\begin{abstract}
Background: This study sought to assess the application of three-dimensional (3D) printing in preoperative planning for anomalous pulmonary venous connection (APVC).

Methods: From November 2017 to January 2019, 17 children diagnosed with APVC were enrolled in this study (total APVC supracardiac type in 10 children, intracardiac type in 2 children, infracardiac type in 1 child, mixed type in 1 child, partial APVC in 3 children). The age was ranged 2 days to 20 months old (median age $1 \mathrm{~m} 5 \mathrm{~d}$ ). Before operation, 3D-printed patient-specific heart models were created based on a cardiac computed tomography (CCT) data set with photosensitive resin materials in stereolithography (SLA) technology. These 3D models were used for presurgical decision making and navigation in the operation room. After surgery, the roles of the 3D models were evaluated with questionnaires.

Results: All 17 children successfully underwent surgeries. 3D heart models accurately demonstrated the malformations, which were all confirmed consistent with surgery findings. The final surgery programs were in accord with presurgical planning. Modeling took $0.5-2 \mathrm{~h}$, with an average of $0.9 \pm 0.4 \mathrm{~h}$. Printing took $2-5 \mathrm{~h}$, with an average of $3.4 \pm 1.2 \mathrm{~h}$. All these children were discharged without adverse events. During follow-up, 2 children suspect of anastomotic stenosis were performed cardiac CT, and 3D printed heart models were created. Results confirmed there was no obvious anastomotic stenosis. Questionnaire results indicate that $3 \mathrm{D}$ printing is a promising technique in clinical practice.
\end{abstract}

Conclusions: 3D printing is beneficial for preoperative planning and post-surgery follow-up in APVC.

Keywords: Anomalous pulmonary venous connection (APVC); congenital heart disease (CHD); surgery; threedimensional printing (3D printing)

Submitted Apr 24, 2019. Accepted for publication Jul 24, 2019.

doi: 10.21037/qims.2019.08.01

View this article at: http://dx.doi.org/10.21037/qims.2019.08.01

\section{Introduction}

Currently, congenital heart disease (CHD) is the most prevalent congenital disability across the world. It affects up to 10 in every 1,000 newborns (1). With the rapid development of modern imaging modalities, surgical and interventional therapies, the mortality and morbidity of CHD have dramatically decreased (2). Despite these developments, complex CHD remains a challenging issue.
Patients with repaired complex CHD and unrepaired cyanotic lesions remain at risk for long-term complications and death (3). Anomalous pulmonary venous connection (APVC) accounts for about $1.5 \%$ of CHD. It means that the pulmonary veins (PVs) are directly or indirectly connected to the right atrium (RA), rather than to the left atrium (LA). If not corrected in time, $80 \%$ of patients die within 1 year of being born. It is one of the few 
Table 1 Characteristics of 17 enrolled children with anomalous pulmonary vein connection

\begin{tabular}{|c|c|c|c|c|c|}
\hline Patient No. & Gender & Age & Main diagnosis & Associated diagnosis & Obstruction \\
\hline 2 & Male & $2 \mathrm{~m} 17 \mathrm{~d}$ & TAPVC (supracardiac) & ASD, RVD, PAH & No \\
\hline 3 & Male & $1 \mathrm{~m} 16 \mathrm{~d}$ & TAPVC (supracardiac) & ASD, PAH & No \\
\hline 4 & Male & $29 d$ & TAPVC (supracardiac) & ASD, PAH, PDA & No \\
\hline 6 & Male & $5 \mathrm{~m} 5 \mathrm{~d}$ & TAPVC (supracardiac) & ASD, PDA, PAH, RVD & No \\
\hline 7 & Male & $9 d$ & TAPVC (supracardiac) & PDA, PAH, ASD, CoA & Yes \\
\hline 8 & Female & $2 d$ & TAPVC (supracardiac) & ASD, PDA, PAH, RVD & No \\
\hline 9 & Male & $23 d$ & TAPVC (supracardiac) & ASD, PAH, RVD & No \\
\hline 12 & Male & $8 d$ & TAPVC (intracardiac) & ASD, PAH & No \\
\hline 13 & Male & $13 d$ & TAPVC (infracardiac) & ASD, RVD, PAH & Yes \\
\hline 14 & Female & $5 \mathrm{~m} 26 \mathrm{~d}$ & TAPVC (mixed) & ASD, PAH & No \\
\hline 15 & Female & $1 \mathrm{~m} 5 \mathrm{~d}$ & PAPVC & CoA, ASD, PAH & No \\
\hline 16 & Male & 1 y $8 \mathrm{~m}$ & PAPVC & ASD, RVD, PAH & No \\
\hline 17 & Male & $10 \mathrm{~m} 28 \mathrm{~d}$ & PAPVC (RSPV, supracardiac) & PLSVC, CSD ASD, VSD & No \\
\hline
\end{tabular}

ASD, atrial septal defect; CoA, coarctation of aorta; CSD, coronary sinus dilation; PAH, pulmonary artery hypertension; PAPVC, partial anomalous pulmonary venous connection; PDA, patent ductus arteriosus; PLSVC, persistent left superior vena cava; RVD, right ventricle dilation; RSPV, right superior pulmonary vein; TAPVC, total anomalous pulmonary venous connection; VSD, ventricular septal defect.

cardiovascular malformations that require surgical repair in infancy and young childhood (4). The imaging modalities applied for APVC include echocardiography, cardiac computed tomography (CCT), and cardiac magnetic resonance (CMR). An exact demonstration of the anatomy of the pulmonary venous connections and assessment of cardiac and extracardiac structures are imperative for proper presurgical planning.

Nevertheless, these images are still limited to twodimensional (2D) display, and cardiologists need to reconstruct the real anatomy in their mind. Indeed, PVs are located behind the heart, making their detection difficult for optimal viewing during surgery. Three-dimensional (3D) printing can provide physicians the ability to vividly understand the anatomical malformations of complex CHD and optimize the preoperative planning $(5,6)$. However, to our knowledge, the clinical value of $3 \mathrm{D}$ printing in preoperative planning for APVC is still lacking. In this study, we sought to investigate the roles of $3 \mathrm{D}$-printed patient- specific heart models in preoperative planning for APVC.

\section{Methods}

\section{Study population}

This study was approved by the institutional review board and informed consent was acquired. From November 2017 to January 2019, 17 children diagnosed with APVC who underwent surgical treatment were enrolled in this study. Their ages ranged from 2 days to 20 months (median age of 1 month and 5 days). The weight range was $2.8-11.2 \mathrm{~kg}$ (median weight of $3.8 \mathrm{~kg}$ ). The diagnoses included total APVC (TAPVC) supracardiac type in 10 children, intracardiac type in 2 children, infracardiac type in 1 child, mixed type in 1 child, and partial APVC (PAPVC) in 3 children. The details of associated malformations and pulmonary venous obstructions of these included children are displayed in Table 1. 

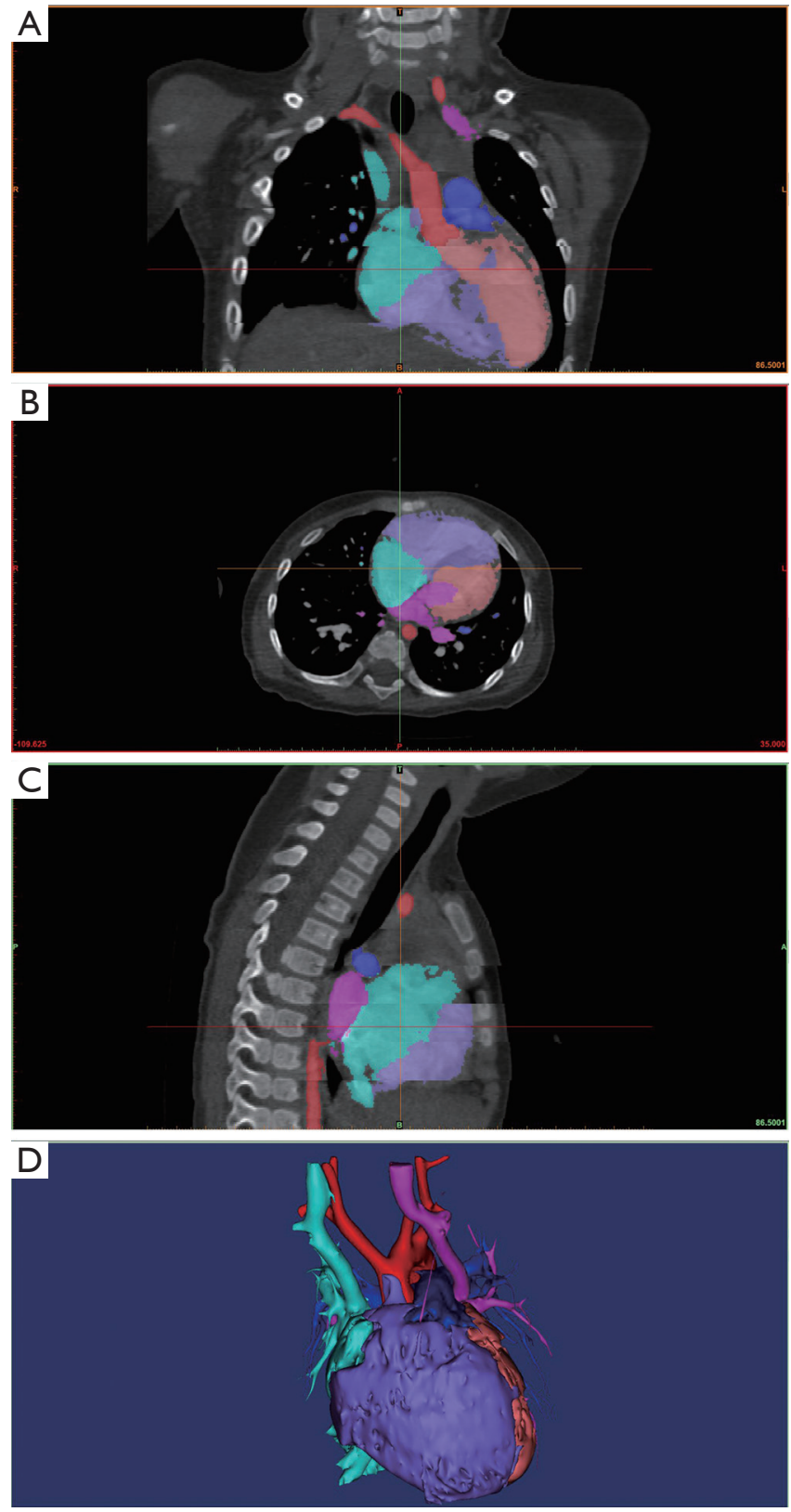

Figure 1 Image segmentation and postprocessing in Mimics 19.0 software. The colored masks were segmented for 3D modeling. (A) Coronal plane; (B) transverse plane; (C) Sagittal plane; (D) 3D modeling. Green, superior vena cava and right atrium; purplish red, pulmonary veins and left atrium; purple, right ventricle; orange, left ventricle; red, aorta; dark blue, pulmonary artery.

\section{Cardiac CT protocol}

All CT examinations were performed with retrospective electrocardiography (ECG)-gated, helical acquisition, with a collimation of 64 detectors $\times 0.625 \mathrm{~mm}$, using a CT scanner (GE Healthcare, USA). The rotation time was $0.35 \mathrm{~s}$, and the pitch was approximately 0.2 . Tube voltage was $100 \mathrm{kV}$. The scanning areas spanned from the superior border of the chest to the diaphragm or middle abdomen in infracardiac TAPVC. The field of view (FOV) was $250 \mathrm{~mm} \times 250 \mathrm{~mm}$, the matrix size was $512 \times 512$, and slice thickness was $0.625 \mathrm{~mm}$. All children were injected with $2 \mathrm{~mL} / \mathrm{kg}$ of nonionic contrast agent (iopamidol, $370 \mathrm{mg} / \mathrm{mL}$, Bracco, Italy) through a peripheral vein. The injection rate was controlled by an infusion pump within $0.8-2.5 \mathrm{~mL} / \mathrm{s}$, according to the size and stability of the intravenous (IV) catheter and the patient's weight. The CT data were exported to the postprocessing workstation (Advantage Windows 4.2, General Electric, Milwaukee, WI, USA) for advanced assessment.

\section{Image segmentation and postprocessing}

The CT image data saved on the disc were opened by RadiAnt DICOM (Digital Imaging and Communications in Medicine) Viewer (64-bit) software and saved on a PC (i57500, 8GB DDR4 2400, GTX 1050 Ti 4GB). The DICOM data saved on the PC were imported into Mimics 19.0 (Materialise HQ, Leuven, Belgium) for 3D modeling. After the DICOM data were imported into Mimics 19.0, the CT Heart reconstruction module and the appropriate threshold were selected to distinguish the blood flow and myocardial tissue in the heart, and thus, a mask was generated. The mask could be identified with a specific color annotation and edited with crop mask command. After the mask was generated, structures such as papillary muscles, muscle bundles, and outflow tracts in the heart chamber were observed, which meant that the contrast agent was unevenly distributed and needed to be manually edited according to the specific situation (Edit instruction), using erasing (Erade), adding (Draw) and other instructions to process the mask. We manually labelled each area according to the left ventricle (LV), right ventricle (RV), LA, RA, aorta (AO), and pulmonary artery (PA) area modules of the CT heart module, and distinguished them with different colors. Special attention was paid to labeling the boundaries of each part. After labeling, a calculated 3D command was selected to generate the solid 3D reconstruction model of the heart, as shown in Figure 1.

Hollowed models were generated based on related solid models and could be hollowed inside or outside direction in 3-Matic 11.0 software (Materialise HQ, Leuven, Belgium). 


\section{$3 D$ printing process}

The $3 \mathrm{D}$ reconstruction models were smoothed and converted into an stereolithography (STL) format file; a solid model was generated and imported into a $3 \mathrm{D}$ printer (ISLA 650, Manufacturer: Shining 3D, Hangzhou, China) for $3 \mathrm{D}$ printing with the following specifications: printing technology, stereolithography (SLA); printing material, photosensitive resin; printing resolution, $0.1 \mathrm{~mm}$. Finally, a personalized 3D heart model was successfully created.

\section{Preoperative planning and evaluation of models}

Preoperative planning was assessed based on the $3 \mathrm{D}$ personalized heart models, medical history, and imaging data by cardiac surgeons and cardiologists. Surgeries were performed in children with surgical treatment indications. The models were sterilized and brought into the operation room for intraoperative navigation. After surgeries, these heart models were evaluated on whether they were of high quality, and whether they could help presurgical planning, reduce unforeseen circumstances, and benefit medical education. An evaluation pertaining to the issues above was conducted via questionnaire by our cardiac surgeons and cardiologists.

\section{Results}

\section{Preoperative planning}

Patient-specific heart models of these children with APVC were successfully created. Modeling and postprocessing took $0.5-2 \mathrm{~h}$, with an average of $0.9 \pm 0.4 \mathrm{~h}$. The printing process took $2-5 \mathrm{~h}$, with an average of $3.4 \pm 1.2 \mathrm{~h}$. All 17 children underwent surgeries successfully without perioperative events and were discharged without adverse events. The malformations demonstrated by the $3 \mathrm{D}$ models were consistent with intraoperative observations, and presurgical planning was in line with real surgery programs. These heart models could be sterilized and brought into the operating room for surgery navigation. These $3 \mathrm{D}$ models greatly assisted the presurgical planning for APVC surgery and were of great clinical value from our experience.

Four cases (No. 1, 5, 9, 10) with TAPVC supracardiac type are displayed in Figure 2. The PV merged into a trunk, which was connected to the vertical vein (VV) and flowed into the right-sided superior vena cava (SVC). Finally, the PVs blood completely flowed into the right atrium. For supracardiac-type TAPVC, with the $3 \mathrm{D}$-printed heart model applied for preoperative planning, we determined the incision at the transverse sinus or left and right atrium through the heart, disconnecting the PV from the VV. The main trunk of the PV was anastomosed to the posterior wall of the left atrium. The anastomosis should be large enough to avoid postoperative obstruction.

Two cases (No. 11, 12) were intracardiac TAPVC (Figure 3); the atrial parts of the heart of interest were segmented for further observation. After postprocessing, it could be observed from the 3D models that the PVs were connected to the coronary sinus (CS) opening in the right atrium. For intracardiac TAPVC, the tissue between the CS and the interatrial septum was cut through the right atrial incision, so that the PV and the left atrium would be completely unblocked, and a large atrial septal defect (ASD) would be made. The defect was repaired with the patch, and the CS opening was placed into the left atrium.

Only 1 case (No. 13) with infracardiac TAPVC was included in our study (Figure 4). With 3D models, the relationship between PVs and inferior vena cava (IVC) was delineated by hand. The preoperative planning included disconnecting the $\mathrm{VV}$, and extending the incision to the PV opening through the $\mathrm{VV}$ and the pulmonary venous confluence. The appropriate incision in the atria was selected, and left and right atrial anastomosis or bilateral atrial anastomosis was performed according to the anatomical positional relationship of the pulmonary venous confluence, $\mathrm{VV}$ and left and right atrium to avoid postoperative pulmonary venous return obstruction. The interatrial septum was then reconstructed with continuous pericardial suture. The anastomosis was isolated into the left atrium. The surgical steps of complete repair were all consistent with preoperative planning based on 3D heart models.

Three cases (No. 15, 16, 17) were diagnosed with PAPVC, and to observe the abnormal connection further, $3 \mathrm{D}$ printing was applied. The right $\mathrm{PV}$ was connected to the right atrium in 2 cases, and in another case, the right superior PV (RSPV) was linked together with the SVC, as demonstrated in Figure 5. Due to associated ventricular septal defect (VSD) in patient 17, pulmonary hypertension was diagnosed, and it indicated surgery. The PVs were drained into the right atrium or the right $\mathrm{PV}$ flowed into the SVC with ASD and patent foramen ovale; the ASD was expanded and repaired with patches, and the PVs were isolated into the left atrium. Surgery results were in line with presurgical planning.

During follow-up, two cases (No. 3, 4) were suspected 

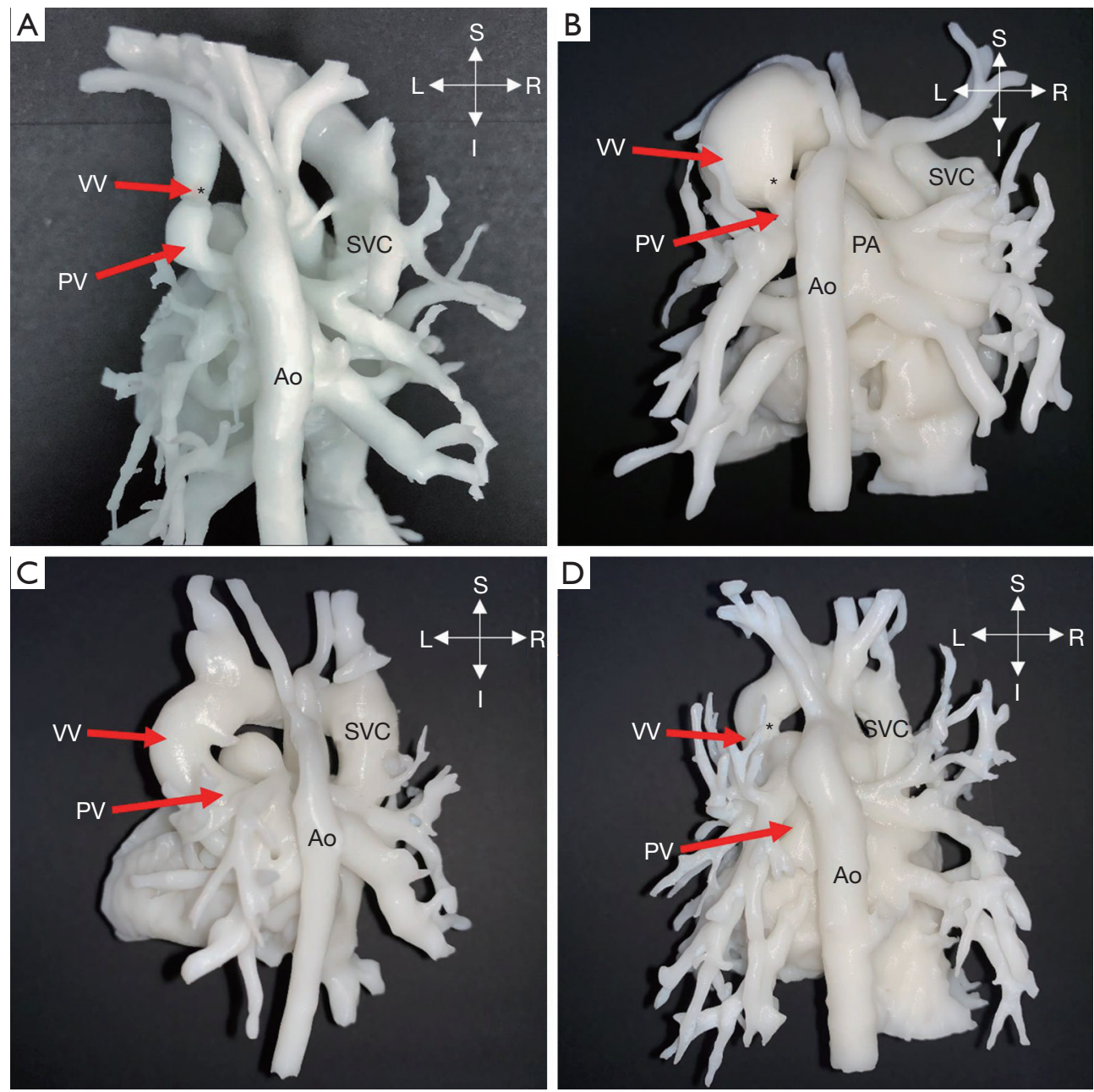

Figure 2 Four cases diagnosed with supracardiac type TAPVC. (A) Refers to patient 1, view from posterior; (B) refers to patient 5 , view from posterior; (C) refers to patient 9, view from posterior; (D) refers to patient 10, view from posterior. *, obstruction exists at the junction of the common pulmonary venous confluence to the left-sided vertical vein (VV). Ao, aorta; PA, pulmonary artery; PV, pulmonary vein; SVC, superior vena cava. Orientation labels: I, inferior; L, left; R, right; S, superior.

of PV stenosis with abnormal echocardiographic parameters and underwent cardiac CT examinations in order to determine whether there was a PV stenosis. We segmented the area of concern and printed heart models, which are displayed in Figure 6. Both blood volume and hollow models were applied to demonstrate the extracardiac and intracardiac structures. The thickness of hollowed models could influence the impression of obstruction, and as a result, during postprocessing, an outside hollowed model was created to avoid the impact on obstruction. The $3 \mathrm{D}$ models showed that there was no visible $\mathrm{PV}$ stenosis in the first case. $\mathrm{PV}$ opening can be observed in the left atrium in Figure 6C. The 3D models in Figure $6 D$ verified the stenosis of the left superior PV (LSPV) of the second case.

\section{Model evaluation}

Evaluation of these models was conducted by cardiac surgeons and cardiologists in regards to the quality of models, the benefit for presurgical planning, the reduction of unforeseen events, and the facilitation of medical education. Thirty-seven physicians in our heart center finished the questionnaires. Results are summarized in Figure 7. 

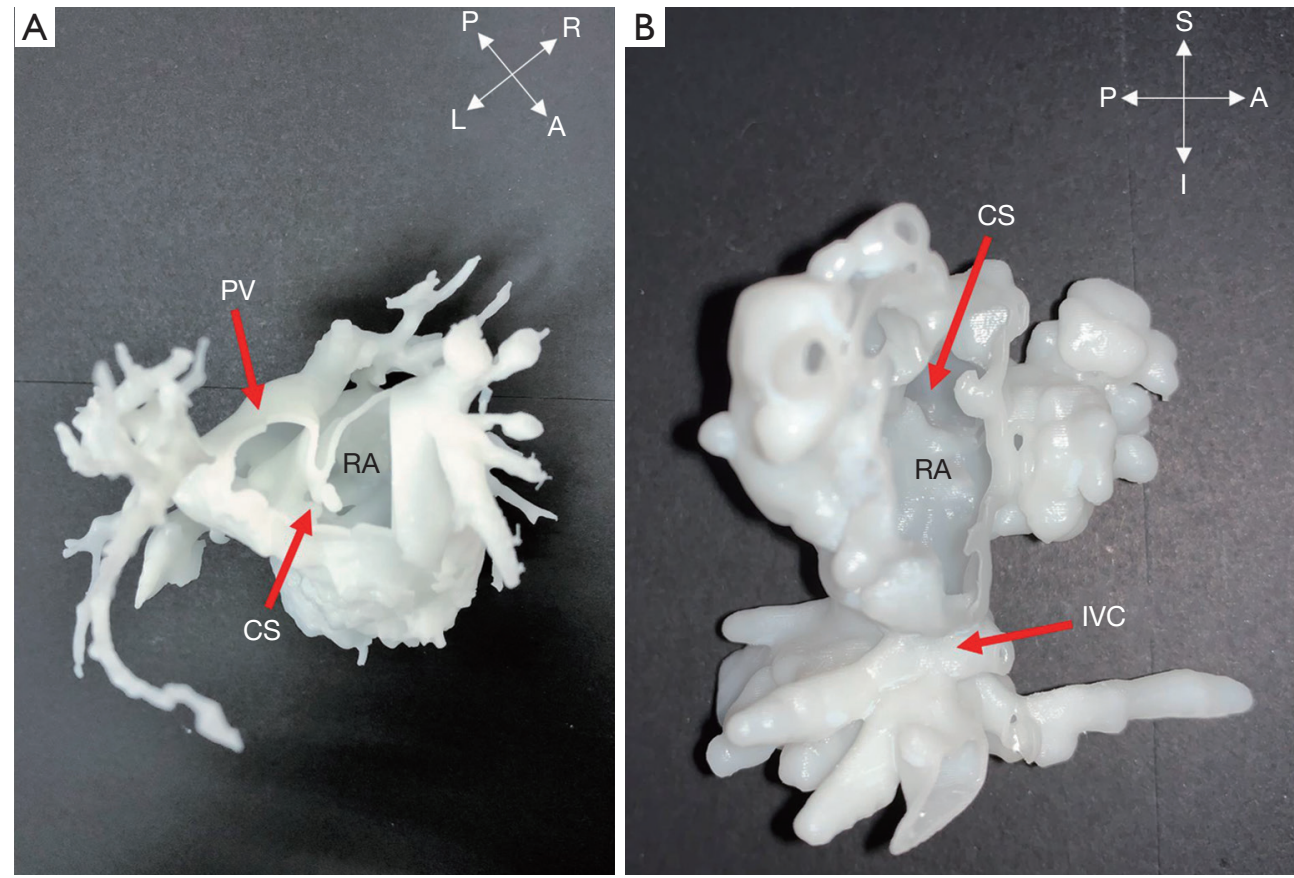

Figure 3 Two cases diagnosed with intracardiac type TAPVC. Part of the hollowed heart model was segmented to emphasize the PV and RA we focused on. Pulmonary vein through the coronary sinus opening in the right atrium. (A) Refers to patient 11, view from inferior; (B) refers to patient 12 , view from the right. CS, coronary sinus; IVC, inferior vena cava; PV, pulmonary veins; RA, right atrium. Orientation labels: A, anterior; I, inferior; L, left; P, posterior; R, right; S, superior.

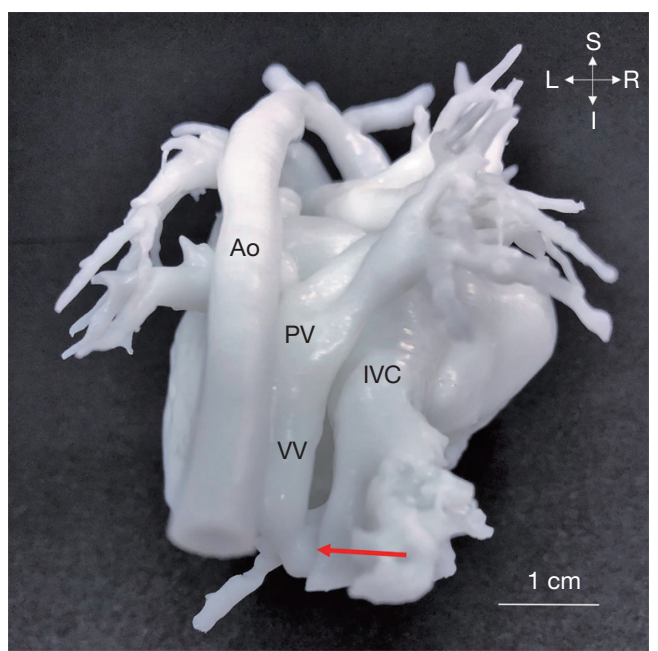

Figure 4 One case (patient 13) diagnosed with infracardiac type TAPVC, view from posterior. The red arrow indicates the junction of the pulmonary vein and the inferior vena cava. Ao, aorta; IVC, inferior vena cava; PV, pulmonary vein; VV, vertical vein; TAPVC, total anomalous pulmonary venous connection. Orientation labels: I, inferior; L, left; R, right; S, superior.

\section{Discussion}

This study demonstrates that $3 \mathrm{D}$ printing is beneficial for the preoperative planning and follow-up of children with APVC. 3D printing transforms the diagnosis and preoperative planning of CHD from the digital platform into a more tangible and vivid physical platform. It can be more easily understood by parents and facilitate communication. The sterilized 3D model can assist in intraoperative navigation and can be preserved for medical education. With these patient-specific heart models, the personalized therapeutic strategy can be made, and the surgery option can be confirmed, which is a reflection of precision medicine. The malformations and preoperative decision making were all consistent with final surgery findings, indicating that $3 \mathrm{D}$ printing will be a promising technology used in cardiac surgery. An international multicenter study found that 3D models accurately replicate cardiovascular anatomy and enhance the understanding of complex CHD. Although it did not change the decision making in most cases, the surgical approach of 19 (47.5\%) 

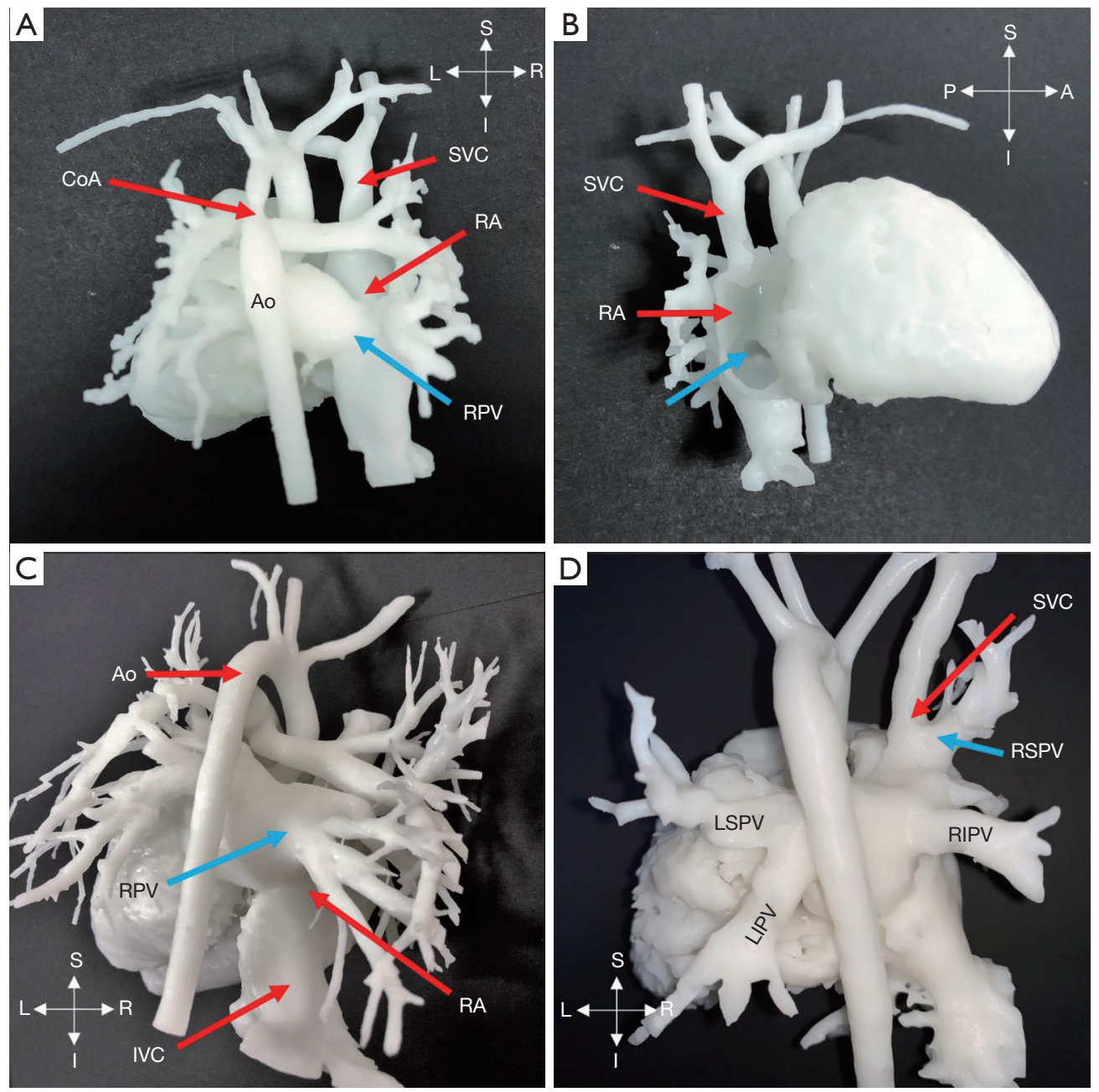

Figure 5 Three cases diagnosed with PAPVC. Images (A,B) both represent patient 15. (A) The blue arrow points to RPV flowing into the right atrium. View from posterior. (B) The blue arrow represents the outlet of RPV, flowing into the right atrium. View from the right. (C) Patient 16 diagnosed with PAPVC; the blue arrow represents RPV flowing into the right atrium. View from posterior. (D) Patient 17. The blue arrow represents RSPV flowing into the SVC. View from posterior. Ao, aorta; CoA, coarctation of aorta; IVC, inferior vena cava; LIPV, left inferior pulmonary vein; LSPV, left superior pulmonary vein; RA, right atrium; RIPV, right inferior pulmonary vein; RPV, right pulmonary vein; RSPV, right superior pulmonary vein; SVC, superior vena cava. Orientation labels: A, anterior; I, inferior; L, left; P, posterior; R, right; S, superior.

selected complex cases were redefined (7). A personalized 3D-printed heart model can assist CHD diagnosis and treatment, and help doctors to more vividly understand the heart anatomy of children with CHD (8-10).

To our knowledge, this is the first study reporting the application of $3 \mathrm{D}$ printing in preoperative planning for a APVC case series with a large sample size. The results of our study indicate that $3 \mathrm{D}$ printed models can illustrate the anatomical malformations and the relationship with other structures, which were confirmed by our surgery observation. Hence, these personalized models are promising tools in preoperative planning for complex CHD. Through image segmentation and postprocessing, intracardiac structures malformations such as VSD and ASD can be evaluated, and the diameter, location, and relationship with great vessels can also be assessed.

However, $3 \mathrm{D}$ printing in APVC is rarely reported. APVC is a rare but more severe CHD. TAPVC accounts for $1-2 \%$ of all cases of major CHD (11). It is essential to observe the positional relationship between the PVs opening and 

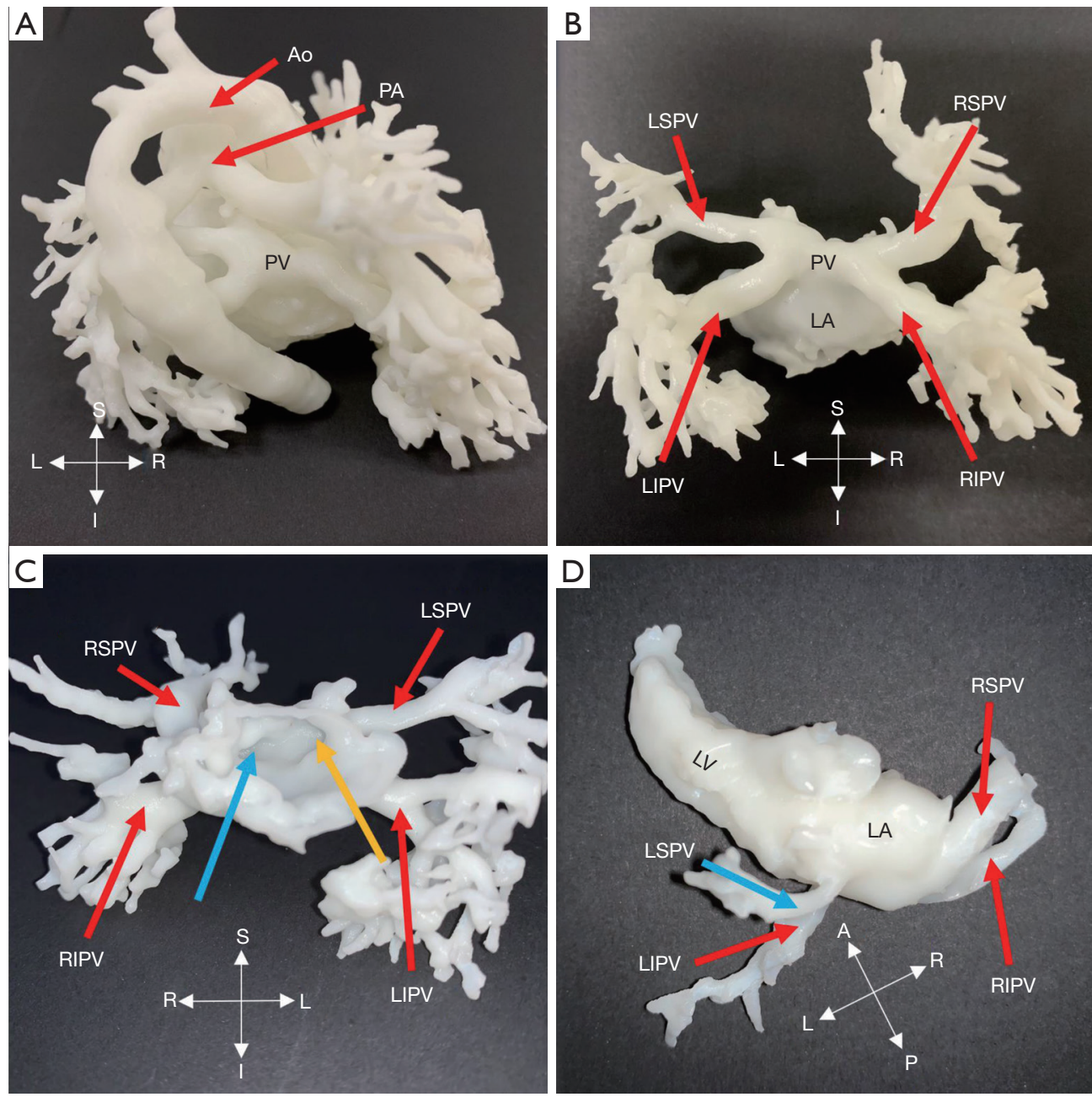

Figure 6 Two cases underwent cardiac CT examination during follow-up. (A,D) Blood volume; (B,C) hollowed models. Images (A,B,C) represent patient 3 after repaired supracardiac TAPVC. (A,B) view from posterior; (C) view from anterior. (C) The blue and yellow arrows represent the opening of the right pulmonary vein and left pulmonary vein. There is no anastomotic stenosis. (D) Superior view of patient 4 after repaired supracardiac TAPVC. Stenosis of LSPV (blue arrow) is shown compared to other pulmonary vein branches. Ao, aorta; LA, left atrium; LIPV, left inferior pulmonary vein; LSPV, left superior pulmonary vein; PA, pulmonary artery; PV, pulmonary vein; RIPV, right inferior pulmonary vein; RSPV, right superior pulmonary vein. Orientation labels: A, anterior; I, inferior; L, left; P, posterior; R, right; S, superior.

the atria before the operation. With the assistance of 3D-printed models, the relationship between PV and atria could be fully understood. A reasonable surgical plan could be implemented according to the information provided by 3D models. Accurate differentiation and characterization of these pulmonary venous anomalies are imperative for treatment planning (12). Due to the limited intraoperative FOV, it is often necessary to detect them during surgery. The 3D-printed heart model can accurately visualize the malformations and thereby reduce unforeseen events
$(10,13)$. The outlet of PV in the left atrium could be observed with the 3D heart models in our study, suggesting an advantage over a 2D imaging modality. McGovern reported 3D printing in complex univentricular hearts with abnormal systemic or pulmonary venous drainage (14). Only three were included; these models were invaluable in the preoperative planning for univentricular hearts and venous drainage abnormalities. Also, they found that surgical intervention might have caused significant complications in two of the patients. In addition, 3D printing was found to 


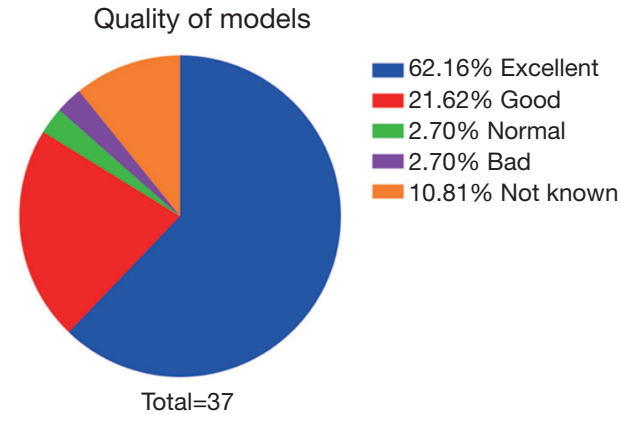

Reduce unforeseen events

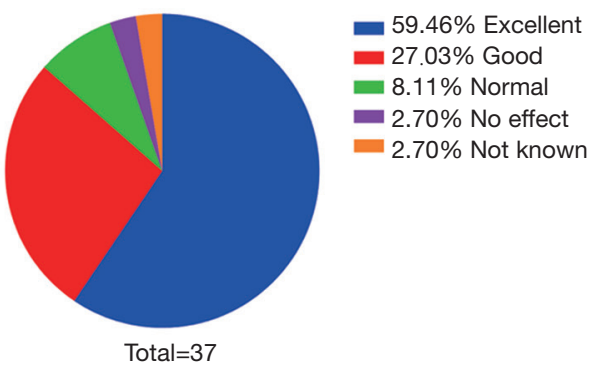

Figure 7 Questionnaire evaluation results.

be able to guide a complex stent angioplasty of pulmonary venous baffle obstruction in a Mustard repair of D-TGA (15). The insight provided by $3 \mathrm{D}$-printed models may help to improve procedural efficiency, decrease radiation exposure, and reduce procedural complications.

Similarly, in our study, during preoperative planning and after surgeries, these models acquired the acceptance of a multidisciplinary team, including cardiac surgeons and cardiologists. Due to the deep position of the PV, they could not be generally observed during surgery. Using 3D models for navigation, it is more efficient to perform surgery: operation time and intraoperative detecting time may be reduced. All children were discharged without complications and adverse events, suggesting $3 \mathrm{D}$ models may lead to superior short-term outcomes. The longterm outcomes of our study still need to be determined. For young inexperienced surgeons, 3D models are useful for medical teaching and education (16) and facilitate communication during clinical consultations (17). All malformations demonstrated by $3 \mathrm{D}$ models were consistent with surgery observations. As the questionnaire results demonstrate, 3D-printed models were of high quality and helped preoperative planning, reduced unforeseen events, and promoted medical education. Undoubtedly, it is a promising tool for future clinical practice.

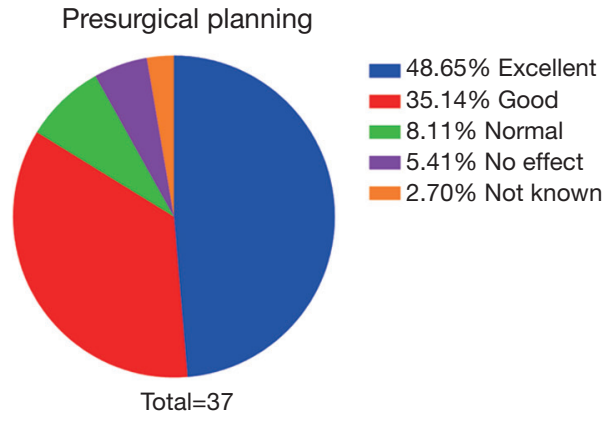

Benefits medical education

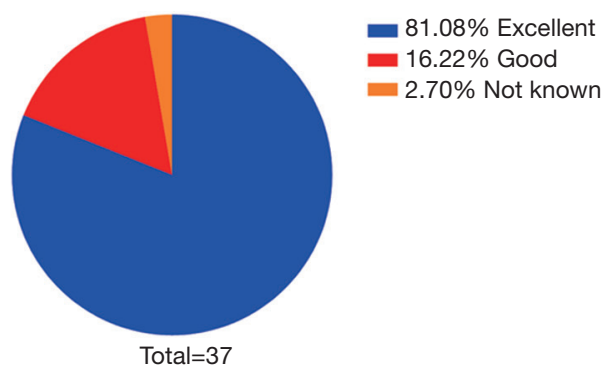

\section{Limitations}

This study has several limitations. First, our 3D printing was based on CT data: the valve structure could not be accurately imaged, so valvular lesions could not be replicated. In the future, a combination of imaging data should be applied for 3D printing. Second, this study is a series of case reports with relatively low levels of evidence and lack of objective quantitative data to confirm the role of $3 \mathrm{D}$ printing technology and whether it can reduce the operation time, bleeding volume, or lead to superior longterm outcomes. It is currently difficult to verify the impact of $3 \mathrm{D}$ printing in a randomized controlled clinical trial. Third, 3D modeling and image segmentation need to be completed manually or semi-automatically and will take a certain amount of time. The $3 \mathrm{D}$ printing process also takes a relatively long time. As a result, 3D printing technology is not practicable in high emergency circumstances. Fourth, $3 \mathrm{D}$ printing of the heart model entails additional costs, and so cost-effectiveness is an issue.

\section{Conclusions}

3D printing is beneficial for presurgical planning in children with APVC. These models can be sterilized and 
brought into operation room for surgical navigation. Unforeseen events can be prevented, while operation time and intraoperative detection time can be reduced. The 3D model not only helps with surgical planning but also can work as a guide for post-surgery follow-up and clarification of outcomes. It will be a promising tool in CHD surgery.

\section{Acknowledgments}

We appreciate the support offered by fellows in the Key Laboratory of 3D Printing Process and Equipment of Zhejiang Province, School of Mechanical Engineering, Zhejiang University.

Funding: This study was funded by the Science Technology Department of Zhejiang Province of China (Grant number: 2016C54006).

\section{Footnote}

Conflicts of Interest: The authors have no conflicts of interest to declare.

Ethical Statement: All procedures performed in studies involving human participants were in accordance with the ethical standards of the institutional and national research committee, and with the 1964 Helsinki declaration and its later amendments or comparable ethical standards. This study was approved by the institutional review board and informed consent was acquired.

\section{References}

1. van der Bom T, Zomer AC, Zwinderman AH, Meijboom FJ, Bouma BJ, Mulder BJ. The changing epidemiology of congenital heart disease. Nat Rev Cardiol. 2011;8:50-60.

2. Hinton RB, Ware SM. Heart Failure in Pediatric Patients With Congenital Heart Disease. Circ Res 2017;120:978-94.

3. Greutmann M, Tobler D, Kovacs AH, Greutmann-Yantiri M, Haile SR, Held L, Ivanov J, Williams WG, Oechslin EN, Silversides CK, Colman JM. Increasing mortality burden among adults with complex congenital heart disease. Congenit Heart Dis 2015;10:117-27.

4. Kaneko Y. Total Anomalous Pulmonary Venous Connection. Kyobu Geka 2015;68:620-3.

5. Giannopoulos AA, Mitsouras D, Yoo SJ, Liu PP, Chatzizisis YS, Rybicki FJ. Applications of 3D printing in cardiovascular diseases. Nat Rev Cardiol 2016;13:701-18.
6. Vukicevic M, Mosadegh B, Min JK, Little SH. Cardiac 3D Printing and its Future Directions. JACC Cardiovasc Imaging 2017;10:171-84.

7. Valverde I, Gomez-Ciriza G, Hussain T, SuarezMejias C, Velasco-Forte MN, Byrne N, Ordoñez A, Gonzalez-Calle A, Anderson D, Hazekamp MG, Roest AAW, Rivas-Gonzalez J, Uribe S, El-Rassi I, Simpson J, Miller O, Ruiz E, Zabala I, Mendez A, Manso B, Gallego P, Prada F, Cantinotti M, Ait-Ali L, Merino C, Parry A, Poirier N, Greil G, Razavi R, Gomez-Cia T, Hosseinpour AR. Three-dimensional printed models for surgical planning of complex congenital heart defects: an international multicentre study. Eur J Cardiothorac Surg 2017;52:1139-48.

8. Anwar S, Singh GK, Varughese J, Nguyen H, Billadello JJ, Sheybani EF, Woodard PK, Manning P, Eghtesady P. 3D Printing in Complex Congenital Heart Disease: Across a Spectrum of Age, Pathology, and Imaging Techniques. JACC Cardiovasc Imaging 2017;10:953-6.

9. Farooqi KM, Saeed O, Zaidi A, Sanz J, Nielsen JC, Hsu DT, Jorde UP. 3D Printing to Guide Ventricular Assist Device Placement in Adults With Congenital Heart Disease and Heart Failure. JACC Heart Fail 2016;4:301-11.

10. Jacobs S, Grunert R, Mohr FW, Falk V. 3D-Imaging of cardiac structures using 3D heart models for planning in heart surgery: a preliminary study. Interact Cardiovasc Thorac Surg 2008;7:6-9.

11. Ferguson EC, Krishnamurthy R, Oldham SA. Classic imaging signs of congenital cardiovascular abnormalities. Radiographics 2007;27:1323-34.

12. Pandey NN, Sharma A, Jagia P. Imaging of anomalous pulmonary venous connections by multidetector $\mathrm{CT}$ angiography using third-generation dual source CT scanner. Br J Radiol 2018;91:20180298.

13. Bhatla P, Tretter JT, Ludomirsky A, Argilla M, Latson LA Jr, Chakravarti S, Barker PC, Yoo SJ, McElhinney DB, Wake N, Mosca RS. Utility and Scope of Rapid Prototyping in Patients with Complex Muscular Ventricular Septal Defects or Double-Outlet Right Ventricle: Does it Alter Management Decisions? Pediatr Cardiol 2017;38:103-14.

14. McGovern E, Kelleher E, Snow A, Walsh K, Gadallah B, Kutty S, Redmond JM, McMahon CJ. Clinical application of three-dimensional printing to the management of complex univentricular hearts with abnormal systemic or pulmonary venous drainage. Cardiol Young 2017;27:1248-56. 
15. Olivieri L, Krieger A, Chen MY, Kim P, Kanter JP. 3D heart model guides complex stent angioplasty of pulmonary venous baffle obstruction in a Mustard repair of D-TGA. Int J Cardiol 2014;172:e297-8.

16. Lim KH, Loo ZY, Goldie SJ, Adams JW, McMenamin PG. Use of 3D printed models in medical education: A randomized control trial comparing $3 \mathrm{D}$ prints versus

Cite this article as: $\mathrm{Xu} \mathrm{J}$, Tian Y, Yin J, Wang J, Xu W, Shi Z, Fu J, Shu Q. Utility of three-dimensional printing in preoperative planning for children with anomalous pulmonary venous connection: a single center experience. Quant Imaging Med Surg 2019;9(11):1804-1814. doi: 10.21037/qims.2019.08.01 cadaveric materials for learning external cardiac anatomy. Anat Sci Educ 2016;9:213-21.

17. Biglino G, Koniordou D, Gasparini M, Capelli C, Leaver LK, Khambadkone S, Schievano S, Taylor AM, Wray J. Piloting the Use of Patient-Specific Cardiac Models as a Novel Tool to Facilitate Communication During Cinical Consultations. Pediatr Cardiol 2017;38:813-8. 